\title{
Investigation of the Erosion Characteristics in the Distribution Parts of Pelton Turbine
}

\author{
L. Han ${ }^{1 \dagger}$, Y. Wang ${ }^{1}$, G. F. Zhang ${ }^{1}$, X. Z. Wei ${ }^{2}$ \\ 1. School of Energy Science and Engineering, Harbin Institute of Technology, Harbin 150001, China \\ 2. State Key Laboratory of Hydro-Power Equipment, Harbin Institute of Large Electrical Machinery, Harbin \\ 150040, China \\ †Corresponding Author Email: hithanlei@hit.edu.cn
}

(Received April 8, 2021; accepted June 22, 2021)

\begin{abstract}
Pelton turbine, as its working principle, can perform in the high head condition especially in the southwest mountainous region in China. When the flow contains silt, it will induce the erosion in the bucket and needle structure which affects the efficiency and furthermore the service life. This paper performs unsteady air-liquidsolid three-phases simulation focusing on the distribution tube and needle parts. Volume of Fluid (VOF) method is used for capturing the free surface flow, at the same time, silt particle is simplified as the sphere particle and simulated through the Discrete Particle Model (DPM). Furthermore, flow detail and erosion information are obtained by conjugating both the former methodologies. Finally, particle size is analyzed in order to investigate the effect of erosion characteristic to the flow passage of Pelton turbine which aims to improve the turbine efficiency.
\end{abstract}

Keywords: Three-phase flow; VOF method; Euler-Lagrange methodology; DPM model.

\section{NOMENCLATURE}

E dimensionless erosion mass

$d_{p} \quad$ particle diameter

$F \quad$ additional force to balance the particle balance

$f(\gamma)$ dimensionless function of the impact angle $\gamma$

$k$ model constant

Re relative Reynolds number

$u \quad$ velocity of the fluid

$u_{p} \quad$ velocity of the particle

$\begin{array}{ll}v & \text { relative particle velocity } \\ v_{n}^{p} & \text { particle impact velocity } \\ \alpha_{w} & \text { liquid volume of fluid } \\ \mu & \text { fluid dynamic viscosity } \\ g & \text { gravity } \\ \rho & \text { density of the fluid } \\ \rho_{p} & \text { density of the particle }\end{array}$

velocity in the distribution and bucket regions reaches high. The erosion is then induced by the continuous friction between silt and the turbine surface. As time goes on, the needle, bucket and distribution tube will be damaged by all these erosion. In the same time, the working life will be shortened and the efficiency will decrease.

In recent years, as the rapid development of Computed Fluid Dynamics (CFD), more and more flow phenomenon in hydraulic turbines is investigated step by step through numerical simulation. However, the flow pattern in the Pelton turbine appears too complex considering the threephase flow and the bucket construction which also increases the difficulty to realize the numerical 
simulation. Secondly, the high velocity passing bucket and needle part induces also the difficulty for experiments. In the same time, limited to the experimental condition, there is rare comparison between numerical simulation and experiments. Thirdly, in the high altitude region, the flow is relatively pure and clean, there is not so much research of the erosion (Luo et al. 2010).

$\mathrm{Xu}$ et al. (2002) performed the solid-fluid flow in the rotating disk with Euler-Lagrange methodology. With Finnie erosion model, the research calculated the erosion rate of the particle to the material and in the same time, obtained both the fluid and particle information to analyze further two phase flow in Pelton turbine. He et al. (2013) also performed CFD investigation in some working point and compared with the theoretical result and shows correspondence between both. This approved that CFD technology is adaptive to the flow in Pelton turbine. Cao et al. (2014(a)) performed numerical simulation focusing on the flow pattern in needle part of the turbine and obtained the erosion characteristics in the region. The flow appears not symmetric and furthermore, erosion is much more evident in the lower part. It seems that the effect of gravity cannot be ignored in the numerical simulation. Guo et al. (2020) using a combination of The Volume of Fluid (VOF) and Lagrangian particle tracking approach to simulate the air-water-sediment flow, followed by the application of Mansouri's model to estimate the erosion. Fundamental analysis of the flow patterns including the vortex structures and the secondary flow on the particle behaviors was carried out to elucidate the asymmetrical erosion distribution on the needle surface. Ge et al. (2020) used the EulerLagrangian method to simulate the air-water-particle three-phase flow with unsteady constant values, and explored the influence of the change of particle diameter and concentration on the abrasion position and wear rate of the impulse turbine, and obtained the particle diameter and the correlation between concentration and wear.

Cao et al. (2014b) investigated the erosion characteristics in the bucket region using the erosion model supposed by Grant and Tabkoff. With the erosion distribution in the inner boundary of the bucket, it is mainly influenced by the diameter, relative velocity and concentration of the particle. Padhy and Saini (2009) performed experiments to investigate mechanism of the erosion in the inner surface of the bucket. Experiments approved material removal from the surface is due to plastic deformation and ploughing of surface. Another series of experiments approved that the size and concentration of the particle are both the key parameters to the erosion. Sugiyama et al. (2004) carried out slurry wear tests, it was found that the volume loss rate of materials at $40 \mathrm{~m} / \mathrm{s}$ is almost constant irrespective of impingement angle between $90^{\circ}$ and $60^{\circ}$, and decreases with a decreased angle. Zehab et al. (2020) used a new non-contact three-dimensional optical scanning technology to study the influence of particles under different working conditions on the abrasion of the Pelton turbine. By measuring the wear depth and the amount of wear of the spear and nozzle, the degree and type of wear were analyzed. Thapa et al. (2012) supposed an empirical modeling of sediment erosion in Francis turbines considering the particle concentration, size, hardness, shape and material characteristics which approves that the erosion is complex enough and should be investigated systematically.

Zeng et al. (2014) simulated the air-fluid-solid three-phase flow with unsteady condition in the needle part of Pelton turbine. The simulation results of the particle flow pattern and erosion prediction in the needle are compared with experimental observation. It approved that the feasibility of the three-phase method in Pelton turbine. However, comparing with the real needle in the power plant, the erosion depth is less predicted. The reason may induced by the simulation without considering the initial cavity phenomenon which can improve the erosion effect. Zhang and Casey (2007) further investigated the flow in distribution tube through experiments by Laser Doppler Anemometry (LDA). A secondary flow generated by the bifurcations in the distributor are still present in the jet in the needle part. They will affect the shape, orientation, and also the topology of the jets and leads a jet center shifting which induces the efficiency decrease. Messa et al. (2019) adopt the Euler-Lagrangian method to simulate the air-solid-liquid flow of a single Pelton turbine nozzle. The stroke of the spray needle is changed, and the abrasion conditions of the nozzle and the spray needle under different spray needle strokes are obtained and the numerical simulation results are highly consistent with this article. Anant et al. (2020) used six different materials to measure the erosion variation in various materials under the same erosive conditions by 3D-scanner and weighing balance method. They found the erosion behavior of all three martensitic steels $(16 \mathrm{Cr}-5 \mathrm{Ni}, 16 \mathrm{Cr}-4 \mathrm{Ni}$ and $13 \mathrm{Cr}-4 \mathrm{Ni}$ ) are similar whereas bronze shows the most erosion for every operating condition. Among the two tested coating materials $(13 \mathrm{Cr}-4 \mathrm{Ni}$ with plasma sprayed $\mathrm{Cr} 2 \mathrm{O} 3$ coating and $13 \mathrm{Cr}-4 \mathrm{Ni}$ with WC-Co-Cr HVOF coating), HVOF coated tungsten carbide shows higher erosion resistance compared to ceramic carbide coating and performed best against hydro-abrasive erosion.

This paper investigates the erosion effect in the distribution parts of Pelton turbine including the distributor, bifurcation tube and also the nozzle part. Three-phase numerical simulation is performed with Euler-Lagrange methodology. According to the simulation results, the flow pattern is analyzed and furthermore, erosion characteristics with different particle status are investigated. Up to now, the research focusing on the three-phase flow in Pelton turbine is relatively rare especially the calculation methodology. This paper performs the first try in this three-phase simulation with the help of limited reference. The erosion phenomenon is analyzed in different particle sizes systematically based on the jet flow pattern which is confirmed by experience in literature. 


\section{MATHEMATICAL MODEL AND NUMERICAL SIMULATION STRATEGY}

\subsection{Mathematical model}

Simulation in this research is performed based on the platform ANSYS Fluent 18. Three-phase flow in distributor tube and nozzle is solved by EulerLagrange methodology in which water and air are defined as continuous phase and particle is defined as the discrete phase. Volume of Fluid (VOF) model is used to define the status of the continuous phase. As the two phases flow is steady, the DPM is added in the three-phase calculation. Then, COUPLE algorithm is carried out to couple particle with the other two phases during the final calculation.

VOF is one of the most used method to capture the interface between two phases in the fixed Euler mesh system. During the iteration process, different components are calculated within the same continuous equation, so the components of the continuous phase are recorded then through the fraction, the interface of the water and air is defined so called the free surface. $\alpha_{w}$ is defined as the volume of fluid. If one mesh contains only air, $\alpha_{w}$ equals to 0 ; contritely, if it contains only water, $\alpha_{w}$ equals to 1 . When it contains two phases in one mesh, this is the interface of the water and air. In this research, we define $\alpha_{w}=0.8$ as the interface.

For simulating the flow characteristics of the solid particle, the silt is defined as the spherical structure with different diameters. DPM model is chosen which can relate the continuous phase and discrete phase and furthermore the trajectory of the particle. The control equations are listed as below according to the Fluent User Guide:

$\frac{\mathrm{d} u_{p}}{\mathrm{~d} t}=F_{D}\left(u-u_{p}\right)+\frac{g\left(\rho_{p}-\rho\right)}{\rho_{p}}+F$

where, $F_{D}\left(u-u_{p}\right)$ is the unit particle drag force, $\mu$ (Pa.s) is the fluid dynamic viscosity, $u_{p}(\mathrm{~m} / \mathrm{s})$ is the velocity of the particle, $u(\mathrm{~m} / \mathrm{s})$ is the velocity of the fluid, $\rho_{p}\left(\mathrm{~kg} / \mathrm{m}^{3}\right)$ is the density of the particle, $\rho$ $\left(\mathrm{kg} / \mathrm{m}^{3}\right)$ is the density of fluid, $F_{D}$ is defined as below:

$F_{D}=\frac{18 \mu}{\rho_{p} d_{p}^{2}} \frac{C_{D} \operatorname{Re}}{24}$

where, $C_{D}$ presents the drag coefficient, $R e$ is the relative Reynolds number which defined as $\mathrm{Re}=\rho v d_{p} / \mu \cdot v(\mathrm{~m} / \mathrm{s})$ presents the relative velocity between particle and fluid, $d_{p}(\mathrm{~m})$ is the particle diameter.

In the Eq. (1), $F$ is defined as the additional force to balance the particle force. One part of the force is virtual mass for which can be defined as
The other part of the force is induced by the velocity gradient in the flow field:

$F=\left(\frac{\rho}{\rho_{p}}\right) u_{p} \nabla u$

Erosion is calculated by the Finnie model scripted in Fluent, the erosion rate is defined as:

$E=K V_{p}^{n} f(\gamma)$

where $E$ is the dimensionless erosion mass, $k$ is a model constant, $V_{p}^{n}$ is the particle impact velocity, and $f(\gamma)$ is a dimensionless function of the impact angle $\gamma$. For metals, the value of the exponent $n$ is generally in the range 2.3 to 2.5 radians. Finnie's model of erosive wear relates the rate of wear to the rate of kinetic energy of impact of particles on the surface using the following functions:

$$
f(\alpha)=\left\{\begin{array}{ccc}
\frac{1}{3} \cos ^{2} \alpha & \text { for } & \alpha>18.5^{\circ} \\
\sin (2 \alpha)-3 \sin ^{2} \alpha & \text { for } & \alpha<18.5^{\circ}
\end{array}\right.
$$

Cao et al. (2020) successfully simulated the air-solid flow in governing valve based on Euler-Lagrange method and Finnie erosion model. The DPM was used to simulate the particle flow, and the Eulerian conservation equation was used to solve the continuous phase. Compared with the reference data, the variation trend of relative flow coefficient is consistent, and the error is less than $5 \%$. Therefore, the simulation results have a good accuracy in this paper.

\subsection{Simulation strategy and boundary condition}

The simulation domain of this research is distribution tube and nozzle flow passage in a Pelton turbine. The complete calculating domain is figured in Fig. 1. In the same time, a column with diameter of $6 d$ and $5.3 d$ length outside the nozzle is added as the air domain as shown in Fig. 1. $d$ value in this figure represents the outlet let diameter of the nozzle structure.

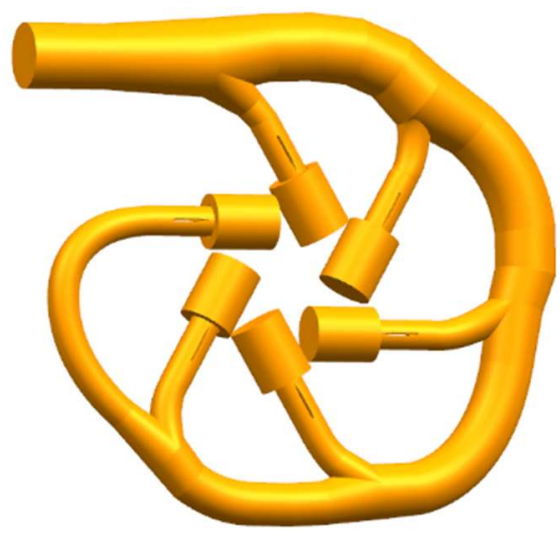

Fig. 1. Global computational domain.
$F=\frac{1}{2} \frac{\rho}{\rho_{p}} \frac{\mathrm{d}}{\mathrm{d} t}\left(u-u_{p}\right)$ 


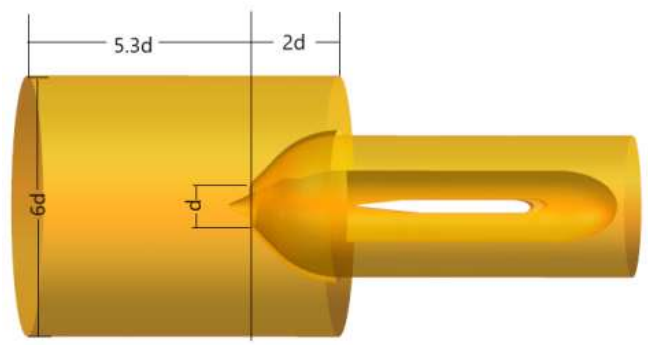

Fig. 2. Computational domain of nozzle (The flow direction is towards left, nozzle outlet diameter $d=49.42 \mathrm{~mm}$ ).

The calculation domain is meshed by the structured mesh as hexahedron blocks. The near wall region and the air region are designed with denser mesh. The entire mesh is shown in Fig. 3a) which has 8.2 million elements in other word 7.9 million nodes in the flow domain. Figure $3 b$ ) shows the detail of the structured mesh of the zooming zone in Fig. 3a).

The turbine is designed in the 80 meter hydraulic head with inlet mass discharge of $197 \mathrm{~kg} / \mathrm{s}$ and the fluid is defined as water. Pressure outlet is set as $0 \mathrm{~Pa}$ which is relative to the atmospheric pressure. The initial flow field is fully filled with air. The wall condition is defined as the standard no-slip condition. For the two phases, air is defined as the major phase to make convergence easier. For the closure of the $\mathrm{N}-\mathrm{S}$ equation, RNG $\mathrm{k}-\varepsilon$ model is chosen. The inlet is set to velocity inlet and pressure outlet is set at the end of the nozzle part. The flow direction is marked as the black arrow in Fig. 3(a). Solid particle equaling to $1 \%$ volume flow rate is added from the inlet through surface rejection method. The density is $2650 \mathrm{~kg} / \mathrm{m}^{3}$, three different diameters are chosen as $1 \mathrm{~mm}, 0.5 \mathrm{~mm}$ and $0.1 \mathrm{~mm}$. The inlet and outlet of the particle are set as escape. It means that when existing particles in the domain reach an inlet or an opening, they escape from the domain. Wall condition is set as reflection. Considering the influence of the turbulence to the particle, particle random model is switched on.

\section{FLOW CHARACTERISTICS VERIFI- CATION WITH EXPERIMENTS}

For confirming the calculation availability of the three-phase calculation, two-phase flow of water and air is preliminarily performed. As the structures of the 6 nozzles are same, $6 \#$ nozzle is chosen to be analyzed in the following. First, the jet structure is shown in the Fig. 4 . where the $\alpha=0.8$ is defined as the surface of the jet.

Along the water jet, 6 sections with the same distance between each two are chosen to be analyzed. They are shown in Fig.5. In the same time, vortex of each section are shown and compared in Fig. 6.

In Fig.6, the process of the jet developing along the flow direction is shown clearly. There are several pairs of the vortex near the jet center. These pairs of the vortex are called Dean vortex which are induced
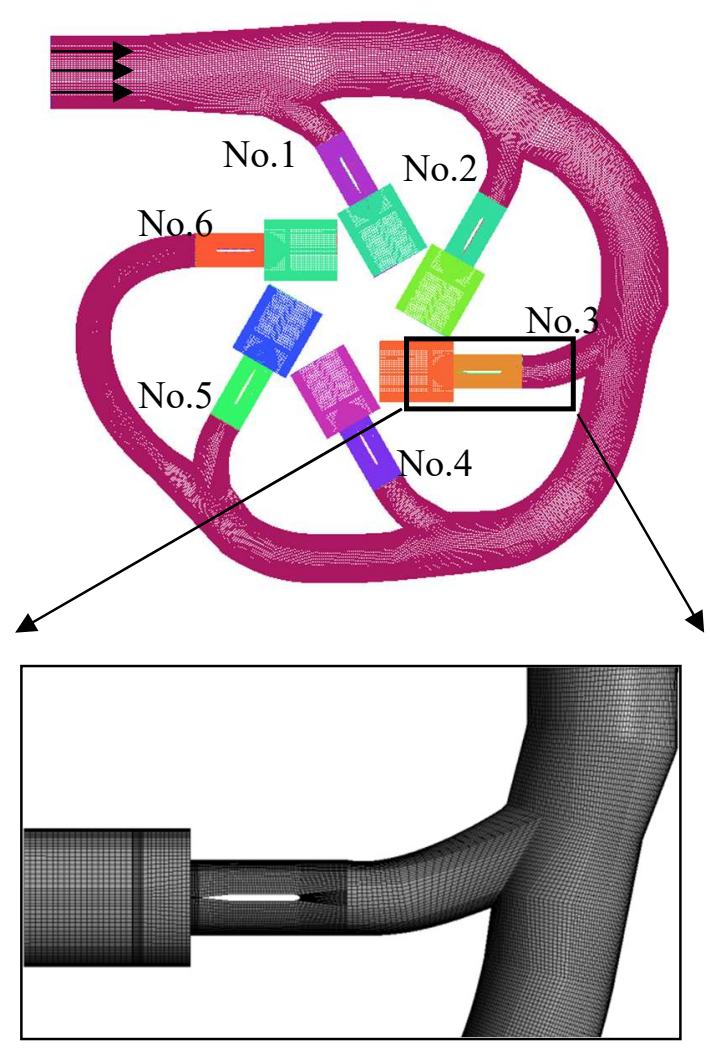

a) up: The entire calculating mesh

b) down: Structure mesh detail of perturbation tube \#3

Fig. 3. Calculating mesh design in fluid domain.

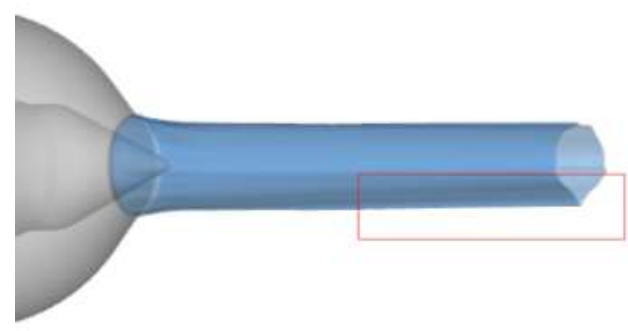

Fig. 4. Shape of jet outside the nozzle.

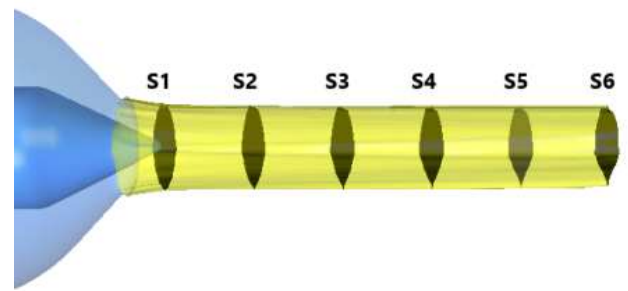

Fig. 5. Selected analyzing section of the jet.

from the distributor and will induce the secondary flow in the pipe (Han et al. 2019). The jet, without the bound of the nozzle boundary, bifurcates by influence of the center vortex. Hence, in Fig.6, the lower boundary deforms outside of the jet round section. This is also observed in the experiment by Zhang and Casay (2007) which is shown in Fig. 7. 
The shape deformation occurs as the jet develops which obtained by the simulation as shown in the red block region in Fig.4.

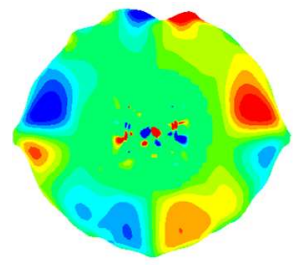

a) Section $\mathrm{S} 1$

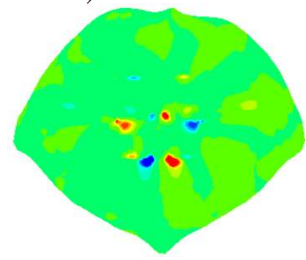

c) Section $\mathrm{S} 3$

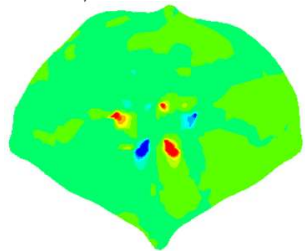

e) Section S5

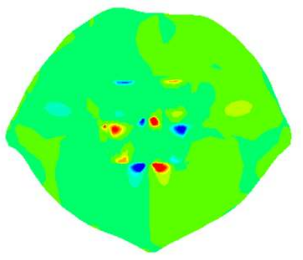

b) Section S2

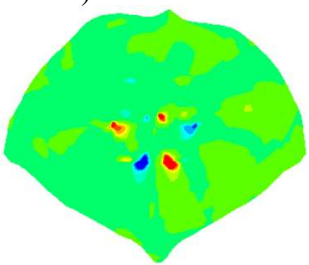

d) Section S4

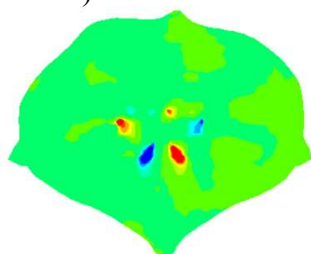

f) Section S6
Velocity.Swirling Normal X

$\left[\mathbf{s}^{\wedge}-1\right]$

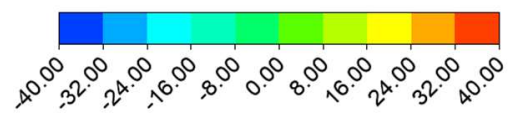

Fig. 6. Velocity vorticity distribution of each section along the jet.

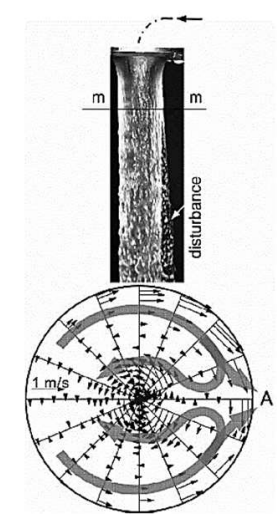

Fig. 7. Jet deformation observed in the experimental from Zhang and Casey (2007).

\section{NUMERICAL SIMULATION RESULTS AND ANALYSIS}

4.1 Particle trajectory and characteristics In this research, the erosion particle is defined as spherical structure for simplification. 3 different sizes of particle are compared together to investigate the particle's influence to the erosion characteristics.
Figure 8 indicates that the flow rate variation of the different particle diameter. This flow rate is monitored in the 6 sections of the nozzle outlets from $1 \#$ to $6 \#$ nozzle.

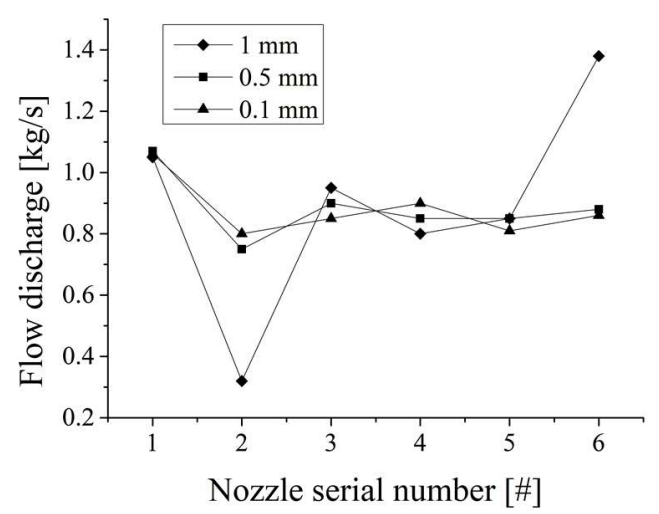

Fig. 8. Particle flow statistics in nozzle outlet.

When the particle is becoming bigger, the distribution of the particle in the bifurcation tube and nozzle parts is less uniform. Furthermore, this is especially evident in the $2 \#$ and $6 \#$ bifurcation tube. When the particle diameter is set to $1 \mathrm{~mm}$, the quantity in $2 \#$ bifurcation tube is far less than the $6 \#$ tube. At the same time, as the diameter becomes smaller, the total amount of particles in $2 \#$ tube increases and decreases relatively in $6 \#$ bifurcation tube. The particle distributions in the other bifurcation tube also become more uniform. Globally, the total amount of particles in 1\# bifurcation is slightly higher than the other 5 tubes. This uniform phenomenon is induced by the different structure of the tube. The curve rate of the upper section of $1 \#$ bifurcation tube is smaller than the other one, so the flow passing 1 \# tube are more fluent than the other. Meanwhile, the other's structure are not strictly same, this also induces uniform flow discharge and furthermore the total amount of particles.

For analyzing the particle detail in different nozzle, the flow streamline and particle path line are compared in Fig. 9. For the case of particle with diameter of $0.1 \mathrm{~mm}$ as Fig. 9(b), the particle trajectory is similar to the streamline in the nozzle. When the flow passes the rib part, the particle direction varies but because of the inertia force of the particle, the particle recovers back to the flow and shows the similar tendency with the flow streamline. This can be seen from Fig. 9(a) and (b). When the diameter becomes larger, the difference between the streamline and particle trajectory occurs. For example, Fig. 9(d) shows the trajectory of the particle diameter of $1 \mathrm{~mm}$. The trajectory is somehow chaotic because the rib structure disturbs the flow pattern and the particle cannot follow the flow as soon as possible. The radial velocity component is larger than the one of small particle. Hence, this induces also the particle trajectory contraction out of the nozzle outlet. Furthermore, as the jet develops, the particle trajectory recovers along the streamline. 


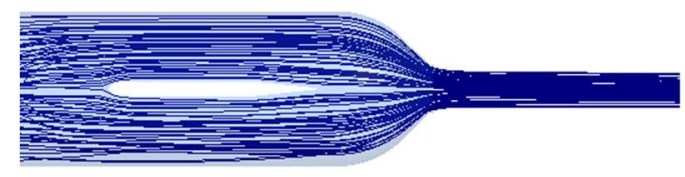

a) Flow streamline in the nozzle

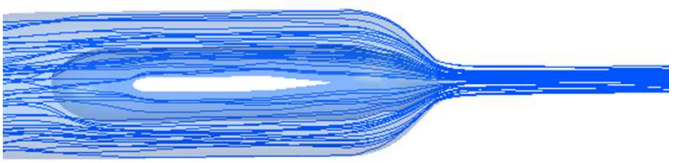

b) Particle trajectories of 6\# nozzle

(Particle diameter $=0.1 \mathrm{~mm})$

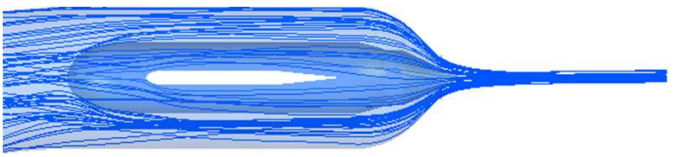

c) Particle trajectories of 6\# nozzle

(Particle diameter $=0.5 \mathrm{~mm}$ )

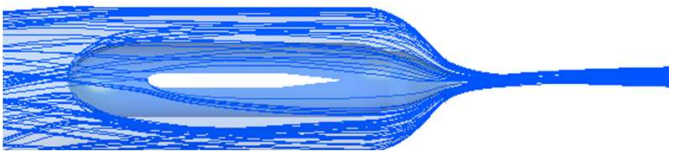

d) Particle trajectories of 6\# nozzle

(Particle diameter $=1 \mathrm{~mm}$ )

Fig. 9. Comparison of streamline and particle trajectories of $6 \#$ nozzle.

As a conclusion, the larger the particle is, the harder for the particle to follow the water flow through the nozzle part of the turbine, and so the bigger difference between the streamline and particle trajectory. This can also explain that why different bifurcation tube has different quantity of particle. Meanwhile, the bigger the particle is, less uniform of the particle distribution in each of the bifurcation tube.

\subsection{Silt erosion characteristics in the flow passage}

In this section, the erosion characteristics are analyzed in the nozzle within different particle size conditions. Most important is to investigate the erosion location and concentration. In Fig.10, all the erosion regions concentrate near the outlet of the nozzle exterior shell.

When the particle diameter equals to $1 \mathrm{~mm}$, the erosion region appears like flake distribution, this reveals that lots of particles attack this region from different directions. Meanwhile, in Fig10 (a), the left and right sides of the nozzle have more serious erosion. Those are the region without influence of the rib. Hence, the rib decreases the erosion of the large size particle in some degree.

Figure 10 (b) shows the erosion of $0.5 \mathrm{~mm}$ size particle. This type of the erosion is different from the

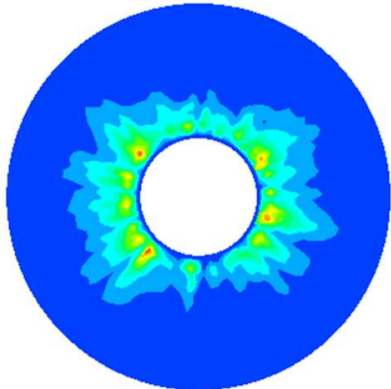

Dpm Erosion Rate Finnie $\quad\left[\mathrm{kg} \mathrm{s}^{\wedge}-1 \mathrm{~m}^{\wedge}-2\right]$

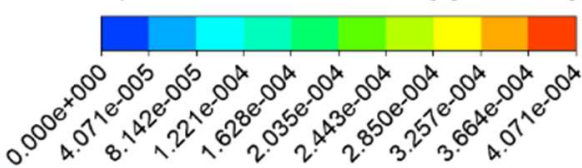

a) article diameter equals to $1 \mathrm{~mm}$
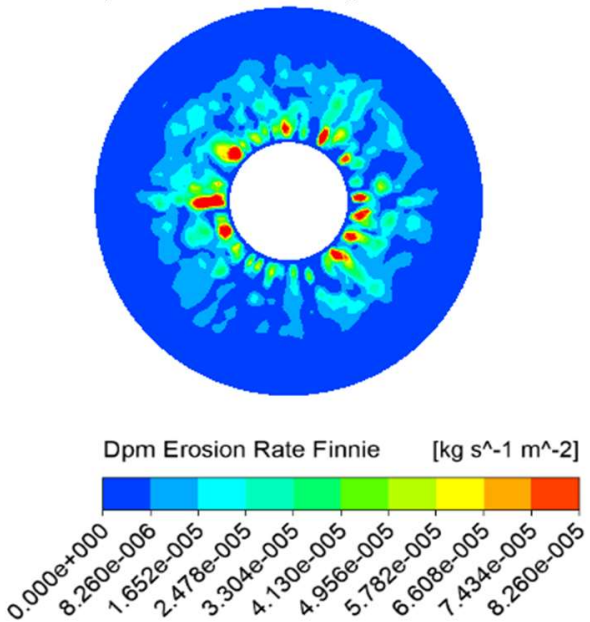

b) Particle diameter equals to $0.5 \mathrm{~mm}$

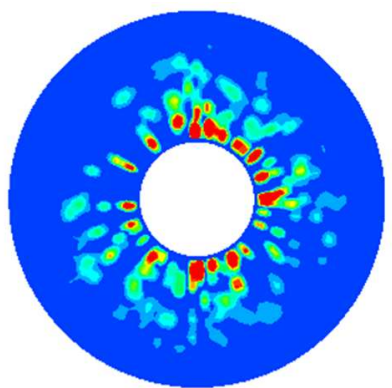

Dpm Erosion Rate Finnie

$\left[\mathrm{kg} \mathrm{s}^{\wedge}-1 \mathrm{~m}^{\wedge}-2\right]$

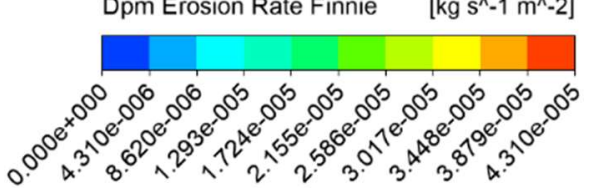

c) Particle diameter equals to $0.1 \mathrm{~mm}$

Fig. 10. Erosion of $6 \#$ nozzle outlet under the condition of various particle sizes.

former one. The erosion region does not appear as the flake region, which is scattered around the nozzle outlet. In the same time, the erosion rate is lower than the one in $1 \mathrm{~mm}$. This can be concluded that different size of the particle has different following performance. Erosion by particles of $0.1 \mathrm{~mm}$ 
diameter is shown in Fig. 10 (c) which is similar to the one of $0.5 \mathrm{~mm}$ diameter.

In the Pelton turbine, the nozzle part includes two parts: on is the outer part which is analyzed in last subsection, the other one is the needle inside to control the nozzle opening which can adjust the flow velocity according to different working conditions.

Evidently, erosion by $1 \mathrm{~mm}$ particle appears different from these by the other two types of particles. Figure 11 (a) shows the erosion distribution by the particle of $1 \mathrm{~mm}$ diameter. The most serious erosion region locates in the head section of the needle and the erosion appears strip structure. It reveals that when the particle collides the needle, the attacking angle between particle and needle is relatively small. Hence, the erosion is induced by the cut and scrape action. In the end section of the needle (left side in the Fig. 11 (a)), punctiform erosion happens. This is caused by the orthographic attack by the particles. Then, there is a region of slight erosion along the body of the needle which called blind erosion area. In this region, the particles escape from the needle with influence of the end of needle.
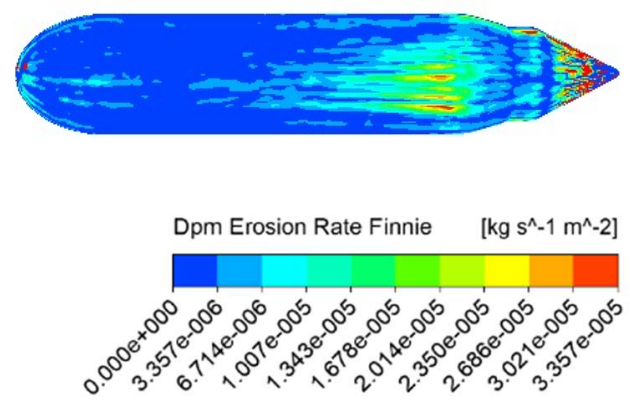

a) Particle diameter equals to $1 \mathrm{~mm}$

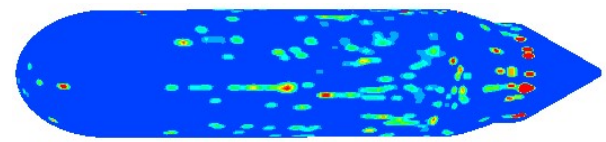

b) Particle diameter equals to $0.5 \mathrm{~mm}$

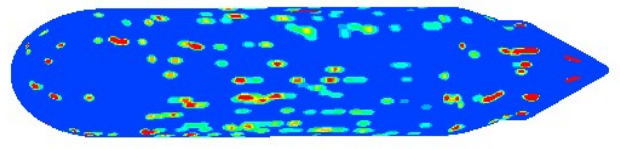

c) Particle diameter equals to $1 \mathrm{~mm}$ Dpm Erosion Rate Finnie $\quad\left[\mathrm{kg} \mathrm{s}^{\wedge}-1 \mathrm{~m}^{\wedge}-2\right]$

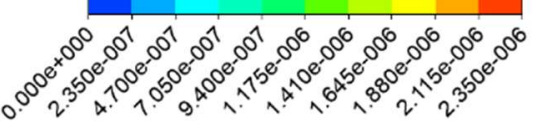

Fig. 11. Erosion of $6 \#$ needle under the condition of various particle sizes.

When the particle size decreases below $0.5 \mathrm{~mm}$ as seen in Fig. 11(b) and (c), the most serious erosion region locates in the neck section of the needle. Meanwhile, there is nearly no erosion in the head section. The no-erosion area on the body is much smaller than the previous condition. Furthermore, for the condition of $0.5 \mathrm{~mm}$ particle, the no-erosion area is even smaller. It concludes that, the smaller the particle is, the better they follow with the fluid along the needle, and the particle distribution is more uniform.

Analyzing the experimental data of Zehab et al. (2020), It can be seen that the closer the nozzle is to the top, the more serious the corrosion, as shown in Fig. 12. In the numerical simulation analysis of this paper, the wear rate from the top to the low end gradually decreases (shown in Fig. 10 and Fig. 11). Therefore, through comparative analysis between Fig.10, Fig. 11 and Fig. 12, the numerical simulation results are highly consistent with the experimental data which verifies the correctness of the numerical simulation results of this paper.
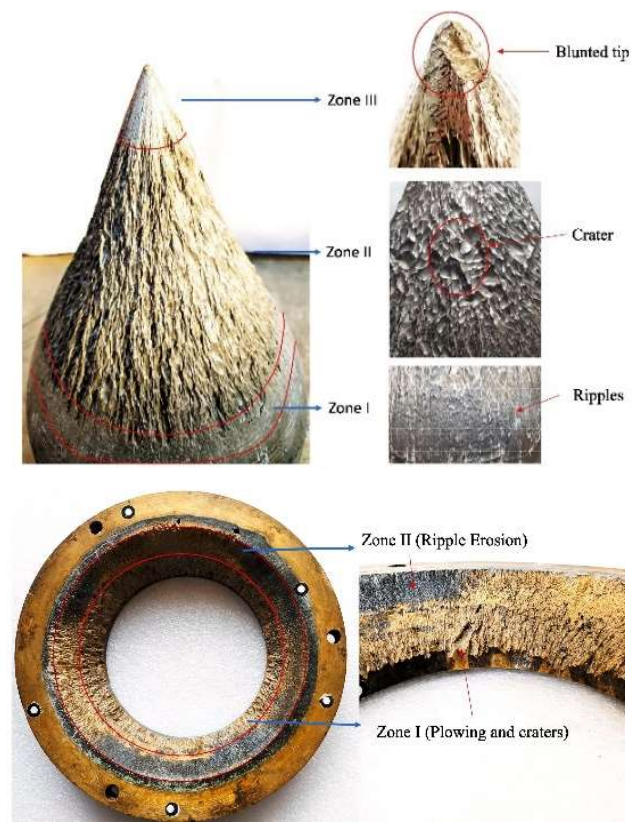

Fig. 12. Wear law of spare and nozzle (Zehab et al. 2020).

\section{CONCLUSION}

This paper performs three-phase flow using numerical simulation focuses on the 6 nozzles Pelton turbine. The working condition is set as below: head is $80 \mathrm{~m}$, normalized nozzle opening is set to 0.55 . Through the numerical simulation, this research achieves three acknowledgement listed below:

(1) In the distribution tube of Pelton turbine, there exists the secondary flow caused by the Dean vortex induced by the tube curving pattern. The vortex develop till the end of the nozzle. Without the bound effect of the wall, the vortex induces the deformation of the jet.

(2) Based on the numerical calculations of coupled VOF and DPM methods, silt defined as spherical particle is then added in the simulation. Results reveal that the bigger the particle is, the harder for the particle to follow up the flow in the passage, more 
difference between the particle trajectory and the flow streamline. Meanwhile, when the particle becomes larger, the distribution of the particle is less uniform in the bifurcation tube and nozzle parts.

(3) The erosion area on the nozzle exterior shell locates mainly near the outlet. Meanwhile, bigger (1 $\mathrm{mm})$ particle induces the flake erosion and the smaller ones $(0.5 \mathrm{~mm}$ and $0.1 \mathrm{~mm})$ bring scattered erosion style. For the erosion on the needle surface, it's mainly located in the head and neck parts. Furthermore, there is also a no-erosion area in the middle section of the needle body.

\section{ACKNOWLEDGEMENTS}

This work was supported by the National Natural Science Foundation of China (Grant No. 51806043).

\section{REFERENCES}

Anant, K. R., K. Arun and S. Thomas (2020). Effect of concentration and size of sediments on hydro-abrasive erosion of Pelton turbine. Renewable Energy 145, 893-902.

Cao, L. H., S. Liu, P. F. Hu and H. Y. Si (2020). The influence of governing valve opening on the erosion characteristics of solid particle in steam turbine, Engineering Failure Analysis 118, 104929 .

Cao, Y., W. W. Song and Q. Huang (2014(a)). Investigation of the two-phase flow in the nozzle of the Pelton turbine. Science and Technology innovation Herald 11(28), 65-66.

Cao, Y., W. W. Song, J. Fu and W. Su (2014(b)). Erosion of buckets of Pelton turbine with sediment. Water Power 40(7), 75-78.

Guo, B., Y. X. Xiao, A. K. Rai, J. Zhang and Q. W. Liang (2020). Sediment-laden flow and erosion modeling in a Pelton turbine injector. Renewable Energy 162, 30-42.

Han, L., X. L. Duan, R. Z. Gong, G. F. Zhang, H. J. Wang and X. Z. Wei (2019). Physic of secondary flow phenomenon in distributor and bifurcation pipe of Pelton turbine. Renewable Energy 131, 159-167.

He, L. P., Q. Y. Wang and W. J. Liu (2013). Numerical simulation in the Pelton turbine. The $19^{\text {th }}$ Symposium on Hydraulic machinery of China 8, 129-136.

Ge, F., J. Sun, Y. Li, D. Wu, Z. Li and H. C. Hua, (2020). Numerical simulation of sand abrasion characteristics of jet mechanism of impulse turbine. Journal of Hydraulic Engineering 51(12), 1486-1494

Luo, J., J. Han, C. H. Wang and J. Zhan (2010) Manufacture situation and tendency of Pelton turbine generator runner. Journal of Chongqing University of Science and Technology (Natural Sciences Edition) 12(03), 103-106.

Messa, G. V., S. Mandelli and S. Malavasi (2019). Hydro-abrasive erosion in Pelton turbine injectors: A numerical study. Renewable Energy 130, 474-488.

Padhy, M. K. and R. P. Saini (2009). Effect of size and concentration of silt particles on erosion of Pelton turbine buckets. Energy 34(10), 14771483 .

Sugiyama, K., S. Nakahama and S. Hattori (2004). Slurry wear and cavitation erosion of thermalsprayed cermets. Wear 258(5), 768-775.

Thapa, B. S., B. Thapa and O. G. Dahlhaug (2012). Empirical modelling of sediment erosion in Francis turbines. Energy 41(1), 386-391.

Xu, Y., Z. Zhang and X. W. Cheng and Y. G. Zheng (2002). Numerical simulation of abrasive erosion in the solid-liquid two-phase rotary flow. Journal of Beijing University of Chemical Technology 29(3), 12-16.

Zehab, U. D. M. and G. A. Huang (2020). Assessment of erosive wear of Pelton turbine injector: Nozzle and spear combination - A study of Chenani hydro-power plant. Engineering Failure Analysis 116, 104695.

Zeng, C. J., Y. X. Xiao and Z. W. Wang (2014). Pelton turbine needle erosion prediction based on 3D Three-phase flow simulation. IOP Conference Series: Earth and Environmental Science 22(5), 22-26.

Zhang, Z. and M. Casey (2007). Experimental Studies of the Jet of a Pelton turbine. Proceedings of the Institution of Mechanical Engineers, Part A: Journal of Power and Energy 221(8), 1181-119. 